\title{
Hepatic schwannoma: A case report and an updated 40-year review of the literature yielding 30 cases
}

\author{
DA-LONG WAN ${ }^{1-3^{*}}$, ZHENG-LONG ZHAI $^{1-3^{*}}$, KUI-WU REN $^{1-3}$, \\ YUN-CHUAN YANG ${ }^{1-3}$, SHENG-ZHANG LIN ${ }^{1-3}$ and SHU-SEN ZHENG ${ }^{1-3}$ \\ ${ }^{1}$ Department of Hepatobiliary and Pancreatic Surgery; ${ }^{2}$ Key Laboratory of Combined Multi-organ Transplantation, \\ Ministry of Public Health; ${ }^{3}$ Collaborative Innovation Center for Diagnosis and Treatment of Infectious Diseases, The \\ First Affiliated Hospital of Zhejiang University, School of Medicine, Hangzhou, Zhejiang 310003, P.R. China
}

Received July 14, 2015; Accepted February 25, 2016

DOI: $10.3892 / \mathrm{mco} .2016 .850$

\begin{abstract}
Hepatic schwannoma is a rare benign disease with a good prognosis. Early diagnosis is difficult due to the absence of specific clinical presentations and its rarity. The present study briefly described a 64-year-old female patient with hepatic schwannoma mimicking intrahepatic cholangiocarcinoma. Furthermore, the clinical data of 30 patients with hepatic schwannoma were also reviewed and analyzed. The mean age of the 30 patients was 51.7 years (range, 21-83 years) and $2 / 3$ were female. All patients in the benign group underwent surgical treatment and survived until the last follow-up, of whom 19 received complete resection and the remaining 1 underwent liver transplantation. However, in the malignant group, only three cases who underwent the surgical resection remained alive at last follow-up. Another seven cases were succumbed to mortality, 4 cases of whom had deteriorated to have no operation opportunity by the time they saw a doctor, and among the remaining three cases with hepatectomy, 1 died of liver dysfunction at 21 days postoperatively, 2 succumbed to recurrences at 18 and 23 months postoperatively. In conclusion, hepatic schwannoma is a rare benign disease with a good prognosis. However, once the malignant transformation occurs, the prognosis is not satisfied. Complete resection is the mainstay for cure and liver transplantation is often necessary.
\end{abstract}

Correspondence to: Professor Shu-Sen Zheng or Dr Sheng-Zhang Lin, Department of Hepatobiliary and Pancreatic Surgery, The First Affiliated Hospital of Zhejiang University, School of Medicine, 79 Qingchun Road, Hangzhou, Zhejiang 310003, P.R. China

E-mail: shusenzheng@zju.edu.cn

E-mail:wzf21sz@163.com

*Contributed equally

Key words: hepatic schwannoma, liver tumor, malignant transformation, liver transplantation

\section{Introduction}

Schwannomas are benign, encapsulated and slowly growing neoplasms which are composed exclusively of Schwann cells. Schwannomas usually occur in the upper extremities, trunk, head and neck, retroperitoneum, mediastinum, and pelvis (1). It has been reported to occasionally occur in the rectum, pancreas, bile duct and stomach; however, schwannomas arising from the liver parenchyma are extremely uncommon. Hepatic schwannoma may cause non-specific clinical symptoms. Since hepatic schwannoma is such a rare finding, a definitive diagnosis by radiological methods is difficult and they are sometimes misinterpreted as liver metastases or primary liver cancer. Surgery is typically required in order to establish the diagnosis and treatment (2).

The present study found only 30 cases reported in the literature to date after carefully excluding those papers which are duplicated and with insufficient clinical data. The present study described a case of hepatic schwannoma and provide a pertinent review of the literature.

\section{Case report}

On March $12^{\text {th }}$ 2015, a 64-year-old Chinese male was referred to The First Affiliated Hospital of Zhejiang University (Hangzhou, China), with complaints of upper abdominal pain for a month. A month ago, the patient suffered from upper abdominal pain without obvious reasons and no chills, no fever, no vomiting, no cough or other symptoms. No family history or obvious evidence of neurofibromatosis was present. The abdominal physical examination revealed no marked findings and all laboratory data were normal, including tumor markers and liver function. The computed tomography (CT) scan (Fig. 1A-C) revealed an $11.5 \times 9.5 \mathrm{~cm}$ mass in the left liver, slightly enhanced in the arterial phase, progressive enhanced in the portal and equilibrium phase, and the left branch of the portal was not visible. The hilar and retroperitoneal area revealed a shadow of multiple lymph nodes. So cholangiocarcinoma was considered initially with the left portal vein branch violation. Magnetic resonance imaging (MRI) (Fig. 1D-F) also revealed an $11.5 \times 9.5 \mathrm{~cm}$ mass, with a low signal on T1-weighted images and higher signal on T2-weighted images. The left 

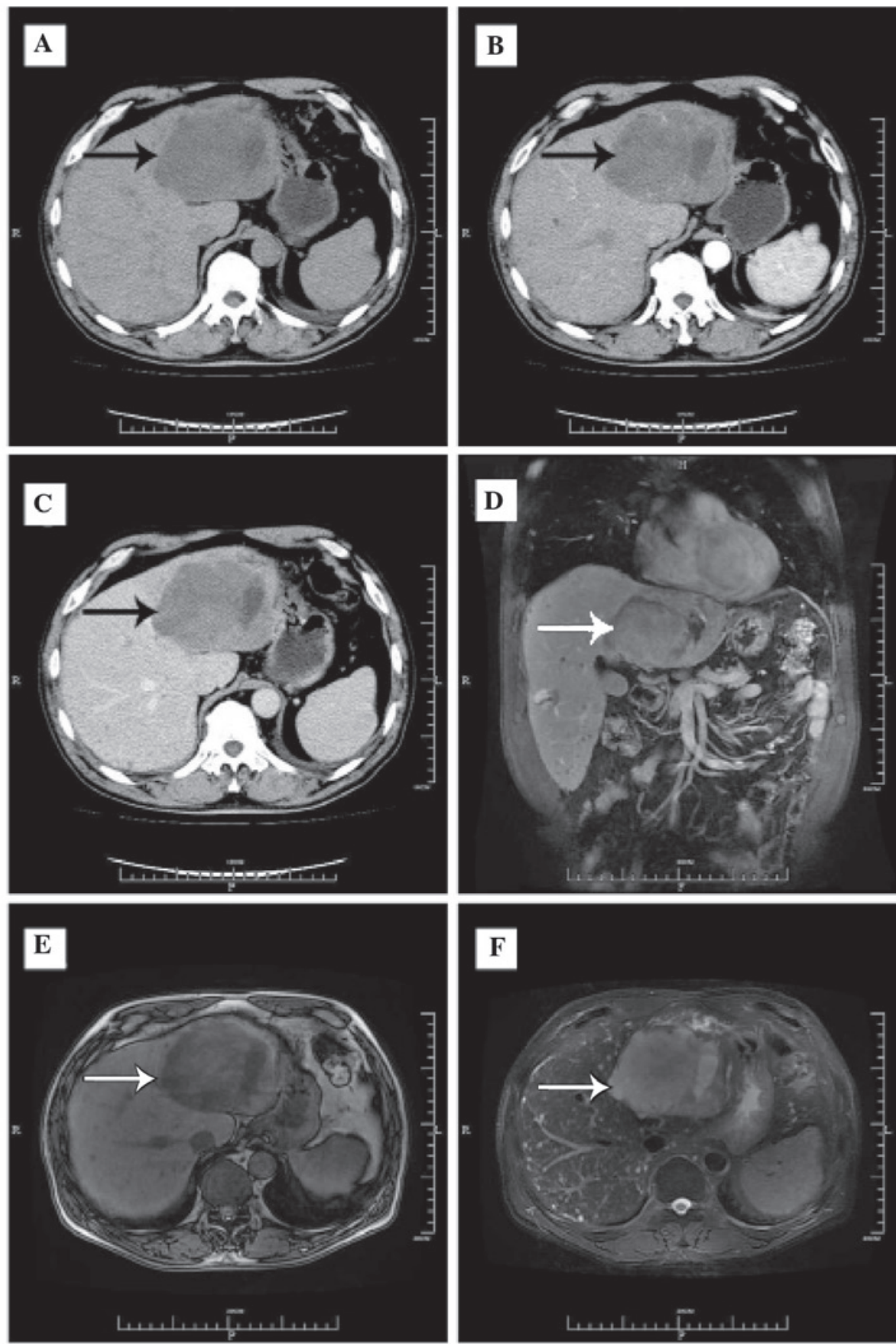

Figure 1. Abdominal CT and MRI findings of the patient. (A) An abdominal CT scan revealed an $11.5 \times 9.5 \mathrm{~cm}$ mass in the left liver (arrow). (B) An enhanced abdominal CT scan revealed a mild heterogeneous enhancement signal (arrow) and a (C) wash-out pattern in the portal phase (arrow). (D) MRI revealed an $11.5 \times 9.5 \mathrm{~cm}$ mass in the sagittal section (arrow), (E) low-signal intensity in T1-weighted axial imaging (arrow) and (F) high signal intensity in T2-weighted imaging (arrow). CT, computed tomography; MRI, magnetic resonance imaging.

portal vein branch was not observed. According to the history and radiographic examination of the patient, surgical resection was selected for diagnosis and cure together by the patient and the treatment team in various departments, including those of Hepatobiliary Surgery, Radiology and Chemotherapy. During the laparotomy, a hard protruding mass with a size of $\sim 11.5 \times 9 \mathrm{~cm}$ was observed on the II, III and IV segment of the liver. Therefore, a left hepatectomy and post-operative detailed histopathological examination was performed, and revealed hepatic benign schwannoma (Fig. 2): cytokeratin (pan)(-), Vimentin(+), CD117(-), S-100(+), Desmin(-), CD34(-) and smooth muscle actin [(SMA)-]. The patient was discharged on postoperative day 14. Until now, no symptoms of recurrence were observed in the patient.

\section{Literature review}

PubMed (https://www.ncbi.nlm.nih.gov/pubmed), MEDLINE (http://webofknowledge.com/MEDLINE), EMBASE (https://www.embase.com), The Cochrane center (https://china. cochrane.org), The Chinese Biology and Medicine Database (http://sinomed.imicams.ac.cn/zh/), The Chinese Wanfang 


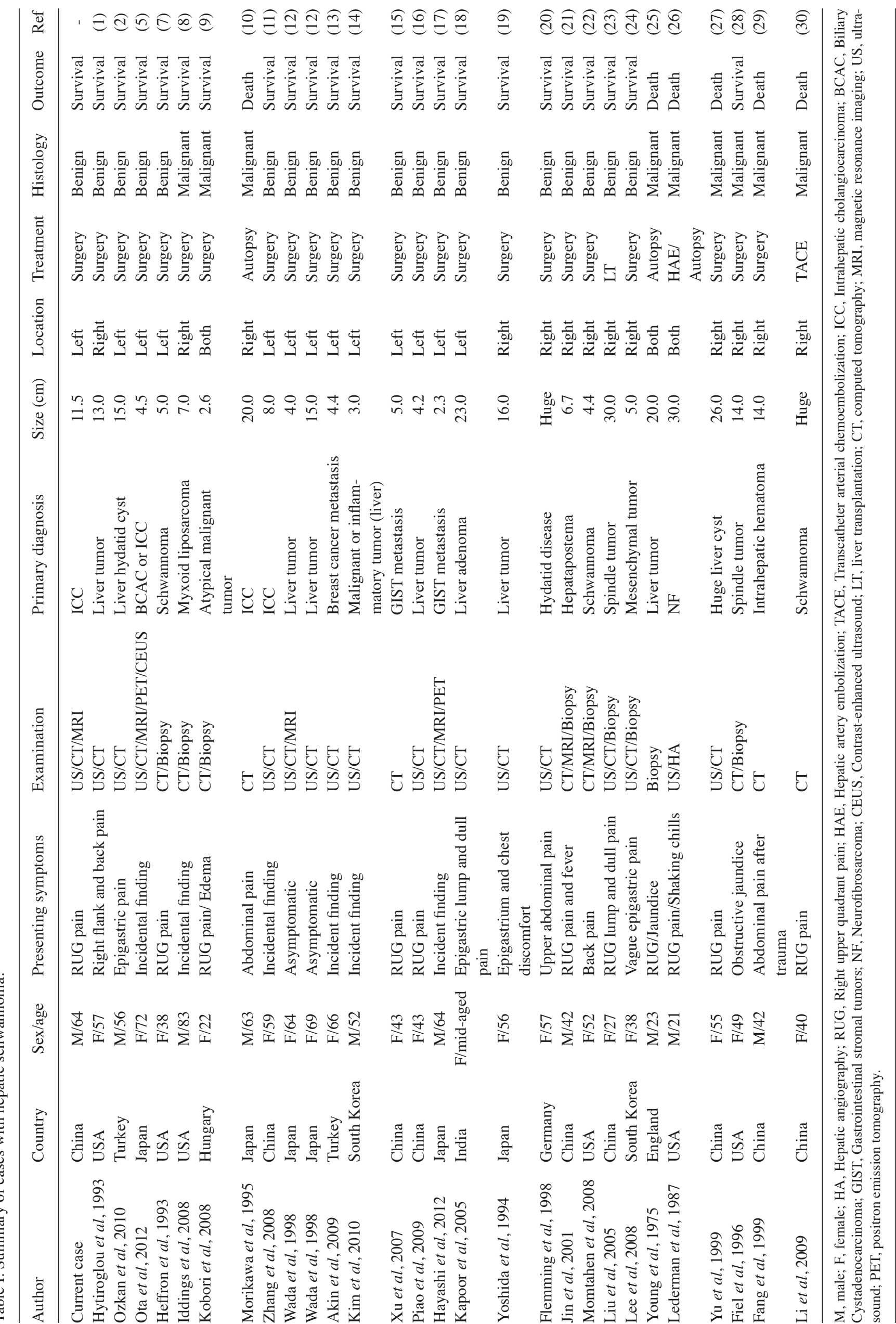



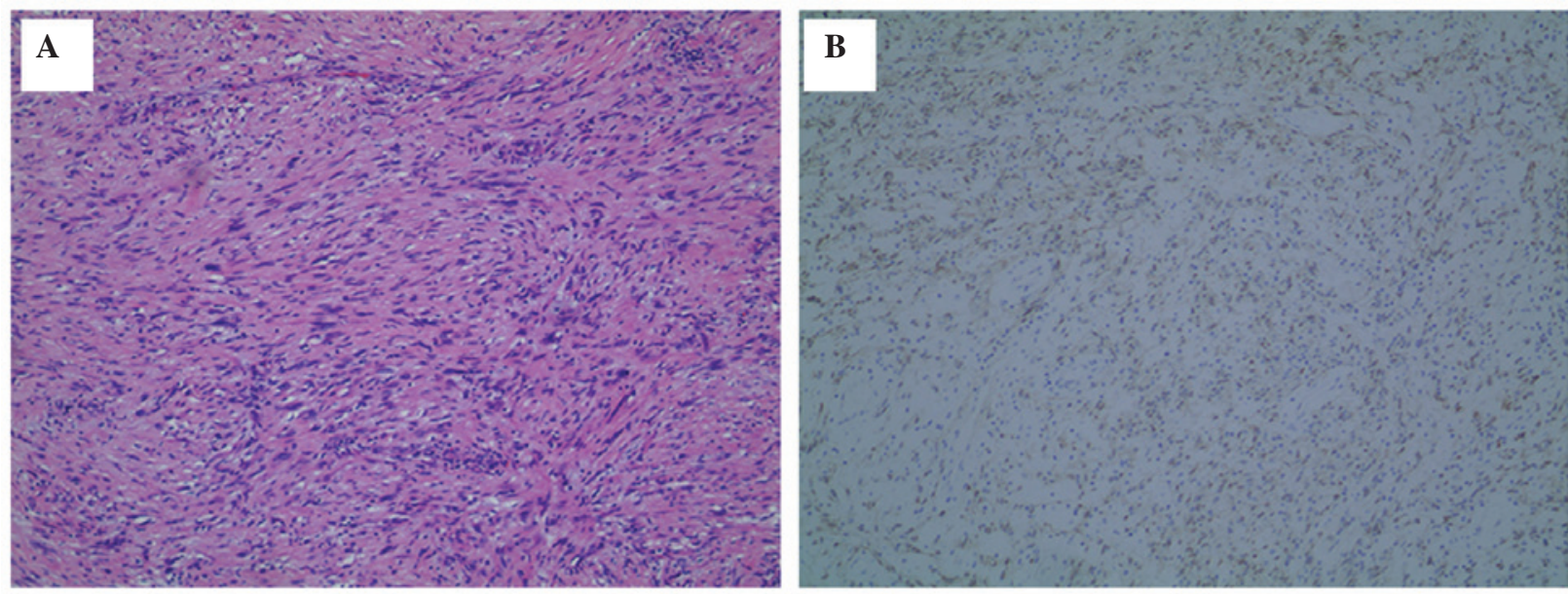

Figure 2. Histological examination of the tumor. (A) Hematoxylin and eosin staining revealed bundles of uniform spindle cells whose elongated nuclei were arranged in a palisading pattern (magnification, x100). (B) Immunohistochemistry revealed that tumor cells were positive for S100 protein (magnification, x100).

Database (http://g.wanfangdata.com.cn) and The China Hospital knowledge Database (http://www.chkd.cnki.net) were searched for cases of primary hepatic parenchyma schwannoma between January 1974 and December 2014. Reports which are duplicated or lacked clinical data were carefully excluded and 30 cases, along with the current case report, were identified (Table I).

\section{Discussion}

Verocay (3) first reported schwannoma as a true neoplasm in 1990. The name schwannoma is often termed differently in primary research, including neurilemmoma, neuroma, neurinoma and nerve sheath tumor. The latest $4^{\text {th }}$ edition of the World Health Organization pathology classification put the peripheral nerve tumors, which included schwannomas, into the soft tissue tumor types, which further demonstrated the understanding of schwannomas (4).

Generally speaking, schwannomas are benign, encapsulated and slowly growing neoplasms, which are composed exclusively of Schwann cells. Schwannomas usually occur in the upper extremities, trunk, head and neck, retroperitoneum, mediastinum and pelvis. It has been reported that occasionally, schwannomas occur in the rectum, pancreas, bile duct and stomach; however, schwannomas arising from the liver parenchyma are extremely uncommon (1). Only 30 cases were found to be reported in the literature to date, following the careful exclusion of those papers which are duplicated and with insufficient clinical data.

Schwannomas occur at all ages, but are most common in individuals aged between 30 and 60-years-old, and the ratio between genders is $\sim 1: 1$ (2). In the present study, the mean age of the 30 patients was 51.7 (range, 21-83 years), which was consistent with the previous report and $\sim 2 / 3$ was female. A total of 18 patients were from Asia, 6 from Europe and 6 from North-America, which may represent that hepatic schwannoma was more common in Asian; however, more evidence is required.

As described previously, the majority of patients were admitted with the complaint of pain or discomfort in the upper abdomen or epigastrium (5). In the present study, 8 patients were asymptomatic, whose liver tumors were found during routine examination or the follow-up when combined with other diseases. A total of 22 patients were symptomatic and symptoms included abdominal pain (91\%), back pain (9\%), chill and fever (9\%), abdominal mass $(9 \%)$, jaundice $(9 \%)$, edema (5\%), and weight loss (5\%).

To differentiate from other primary liver tumors, including hepatocellular carcinoma and intrahepatic cholangiocarcinoma, liver metastases when the patient suffered certain types of malignant tumor, and hydatid disease of the liver when cystic transformation were present, auxiliary examinations including ultrasonography (US), CT, MRI and even positron emission tomography (PET)-CT are required. These techniques can determine tumor position, size, and an appropriate staging of the tumor extension and involved structures, which is of great assistance to diagnose and design an operation scheme. However, since hepatic schwannoma is such a rare finding, a definitive diagnosis by radiological methods is difficult. Liver biopsy may be useful for accurate preoperative diagnosis $(5,6)$.

In the present study, 28 patients received $\mathrm{CT}$ for diagnosis in 30 cases with auxiliary examinations. US, MRI or PET-CT was also selected in certain cases. A total of 9 cases selected preoperative biopsy for diagnosis. Even though the imaging examination is more precise, only 2 patients had the exact diagnosis and other 28 patients were misdiagnosed with other diseases or no confirmed diagnosis.

The final diagnosis of depends on the pathology and immunohistochemistry. Microscopically, a typical schwannoma is composed of Antoni A and Antoni B areas. The Antoni A area is a hypercellular area, which contains a large number of closely packed spindle cells. By contrast, the Antoni B area is a loose myxoid structure with a few sparse cells. The proportion of the two components can shift gradually or change suddenly (7). Cystic degeneration, including calcification or hemorrhage, is often recognized in the Antoni B area, which may result from vascular thrombosis and subsequent necrosis. Immunohistochemical analysis is necessary to distinguish hepatic schwannoma from metastatic gastrointestinal stromal 
tumor types and other soft tissue tumor types. A schwannoma is usually positive for S-100, negative for both CD34 and CD117. However, metastatic gastrointestinal stromal tumors are positive for S-100, and positive for either CD34 or CD117. A leiomyoma would be negative for S-100 and positive for desmin or SMA (8). When the hepatic schwannoma presented with numerous sub-foci around or grown rapidly or associated with a highly-aggressive course of recurrence during follow-up, it almost certainly was the malignant type (9).

In the present statistics, of the 9 malignant schwannoma cases, 1 was semi-malignant and 20 were benign. Although the tumor in certain cases was reported to be S-100 negative, other characteristics of it proved the malignant nature (9). When the patient wants to take a conservative treatment, the pathology and immunohistochemistry of the liver tumor tissue must be performed to exclude the malignant transformation, since survival with observation alone for malignant schwannoma is poor (10).

Since discriminating between a schwannoma and malignant liver neoplasm is difficult, surgery is typically recommended. In order to better observe the difference in the treatment and prognosis between the benign and malignant primary hepatic schwannoma, the 30 patients were divided into two groups. In the benign group, 13/20 cases with liver tumors were located in the left lobe and 7/20 were distributed in the right lobe. The maximal diameter of the tumors reported varied between 2.3 and $30 \mathrm{~cm}$. All patients in the benign group underwent surgical treatment and survived until the last follow-up, of whom 19 received complete resection. The tumor of the remaining patient was too large (the maximal diameter was $30 \mathrm{~cm}$ ) to be removed surgically, so that patient underwent liver transplantation and no recurrence was observed during the follow-up of 4 months. In the malignant group (10 cases, including one semi-malignant case), the location of hepatic schwannoma in 6/10 cases was the right lobe and in 4/10 was both lobes. The size ranged between 2.6 and $30 \mathrm{~cm}$. However, only 3 cases who underwent surgical resection survived at the last follow-up. The other 7 cases succumbed to mortality; 4 patients deteriorated prior to operation, and in the 3 cases with hepatectomy, 1 succumbed to liver dysfunction at 21 days postoperatively and 2 succumbed to recurrences at 18 and 23 months postoperatively. These results suggested that hepatic schwannoma must be resected regardless of the admission diagnosis of benign or malignant, since if schwannomas are benign, surgical treatment can lead to long-term survival and if malignant, enucleation should be oncologically adequate and once radical surgery is impossible, palliative resection is still recommended. To detect recurrence, a CT scan every 3 months for the initial 2 years, every 6 months for 2-5 years and annually thereafter, is generally recommended.

In conclusion, hepatic schwannoma is a rare benign disease with a good prognosis. However, this disease deserves attention with regards to the differential diagnosis of liver tumors. Preoperative diagnosis is highly difficult and complete resection is the mainstay for cure, with liver transplantation sometimes being necessary.

\section{Acknowledgements}

The authors would like to thank the entire staff of the Departments of Hepatobiliary and Pancreatic Surgery,
Pathology, and Radiology at The First Affiliated Hospital of Zhejiang University, School of Medicine (Zhejiang, China) for helpful assistance.

\section{References}

1. Hytiroglou P, Linton P, Klion F, Schwartz M, Miller C and Thung SN: Benign schwannoma of the liver. Arch Pathol Lab Med 117: 216-218, 1993

2. Ozkan EE, Guldur ME and Uzunkoy A: A Case report of benign schwannoma of the liver. Intern Med 49: 1533-1536, 2010

3. Verocay J: Zur Kenntnis der 'Neurofibrome.' Beitr Pathol Anat Allg Pathol 48: 1-69, 1910 (In German).

4. Fletcher CD, Bridge JA, Hogendoorn PC and Mertens: World Health Organization classification of soft tissue and bone tumours. Lyon-IARC Press, 2013.

5. Ota Y, Aso K, Watanabe K,Einama T, Imai K, Karasaki H, Sudo R, Tamaki Y, Okada M, Tokusashi Y, et al: Hepatic schwannoma: Imaging findings on CT, MRI and contrast-enhanced ultrasonography. World J Gastroenterol 18: 4967-4972, 2012.

6. Ortega-Candil A, Rodríguez-Rey C, Cabrera-Martín MN, García García-Esquinas $M$, Lapeña-Gutiérrez $L$ and Carreras-Delgado JL: 18FDG PET/CT imaging of schwannoma mimicking colorectal cancer metastasis. Rev Esp Med Nucl Imagen Mol 32: 332-333, 2013.

7. Heffron TG, Coventry S, Bedendo F and Baker A: Resection of primary schwannoma of the liver not associated with neurofibromatosis. Arch Surg 128: 1396-1398, 1993.

8. Iddings DM, Wright BE and Bilchik A: A rare cause of primary hepatic neoplasm: Malignant peripheral nerve sheath tumor in the age of modern liver surgery. Am Surg 74: 47-50, 2008.

9. Kobori L, Nagy P, Mathe Z, Hartmann E, Doros A, Paku S, Dezso K and Sápi Z: Malignant peripheral nerve sheath tumor of the liver: A case report. Pathol Oncol Res 14: 329-332, 2008.

10. Morikawa Y, Ishihara Y, Matsuura N, Miyamoto $\mathrm{H}$ and Kakudo K: Malignant schwannoma of the liver. Dig Dis Sci 40: 1279-1282, 1995.

11. Zhang L, Yu R and Chen Y: Primary hepatic benign schwannoma: Case report. Chin J Radiol 42: 1068, 2008 (In Chinese).

12. Wada Y, Jimi A, Nakashima O, Kojiro M, Kurohiji T and Sai K: Schwannoma of the liver: Report of two surgical cases. Pathol Int 48: 611-617, 1998.

13. Akin M, Bozkirli B, Leventoglu S, Unal K, Kapucu LO, Akyurek $N$ and Sare M: Liver schwannoma incidentally discovered in a patient with breast cancer. Bratisl Lek Listy 110: 298-300, 2009.

14. Kim YC and Park MS: Primary hepatic schwannoma mimicking malignancy on fluorine-18 2-fluoro-2-deoxy-D-glucose positron emission tomography-computed tomography. Hepatology 51: 1080-1081, 2010

15. Xu L, Zhou X and Qian H: Small bowel stromal tumors associated with hepatic schwannoma: Case report. Chin J Bases Clin General Surg 14: 742, 2007 (In Chinese).

16. Piao G: One Case: Liver Schwannoma. J Pract Radiol 25: 1343, 2009 (In Chinese).

17. Hayashi M, Takeshita A, Yamamoto K and Tanigawa N: Primary hepatic benign schwannoma. World J Gastrointest Surg 4: 73-8, 2012.

18. Kapoor S, Tevatia MS, Dattagupta S, and Chattopadhyay TK: Primary hepatic nerve sheath tumor. Liver Int 25: 458-9, 2005.

19. Yoshida M, Nakashima Y, Tanaka A, Mori K and Yamaoka Y: Benign schwannoma of the liver: A case report. Nihon Geka Hokan 63: 208-214, 1994.

20. Flemming P, Frerker M, Klempnauer J, Pichlmayr R: Benign schwannoma of the liver with cystic changes misinterpreted as hydatid disease. Hepatogastroenterology 45: 1764-1766, 1998.

21. Jin L and Zhou Y: A case of hepatic schwannoma misdiagnosed as liver abscess. Chin J Dig 22: 6, 2002.

22. Momtahen AJ, Akduman EI, Balci NC, Fattahi R and Havlioglu N: Liver schwannoma: Findings on MRI. Magn Reson Imaging 26: 1442-1445, 2008.

23. Liu Y: A case of huge hepatic schwannoma for liver transplantation. Chin network dynamics of infectious diseases: 45-45, 2005 (In Chinese).

24. Lee WH, Kim TH, You SS, Choi SP, Min HJ, Kim HJ, Lee OJ and Ko GH: Benign schwannoma of the liver: A case report. J Korean Med Sci 23: 727-730, 2008. 
25. Young SJ: Primary malignant neurilemmona (schwannoma) of the liver in a case of neurofibromatosis. J Pathol 117: 151-153, 1975.

26. Lederman SM, Martin EC, Laffey KT and Lefkowitch JH: Hepatic neurofibromatosis, malignant schwannoma and angiosarcoma in von Recklinghausen's disease. Gastroenterology 92: 234-239, 1987.

27. Yu C, Li S, Zhang Z and Zhang Z: A case of massive hepatic malignant schwannoma. Chin J Pract Oncol: 308, 1999 (In Chinese).

28. Fiel MI, Schwartz M, Min AD, Sung MW and Thung SN: Malignant schwannoma of the liver in a patient without neurofibromatosis: A case report and review of the literature. Arch Pathol Lab Med 120: 1145-1147, 1996.
29. Fang $X$ and Li C: Primary malignant schwannoma of the liver: Case report. J Hepatopancreatbiliary Surgery 11: 202, 1999 (In Chinese).

30. Tengfei Li, Zhen Wu, Gang Wu and Han X: Hepatic malignant schwannoma in von Recklinghausen's disease: Case report. Chin J CT and MRI 7: 77-78, 2009 (In Chinese). 